\title{
Corona Virus Detection Using Transfer Learning Technique
}

\author{
Sangeetha ${ }^{1}$, Senthil Prabha $\mathrm{R}^{2}$, Ravitha Rajalakshmi ${ }^{3}$ and Srilam $\mathrm{K}^{4}$ \\ \{bsg.it@psgtech.ac.in ${ }^{1}$, rsp.it@psgtech.ac.in ${ }^{2}$, nrr.it@psgtech.ac.in ${ }^{3}$ sksrilam@gmail.com ${ }^{4}$ \} \\ ${ }^{1,2,3}$ Assistant Professor (Senior Grade), Department of Information Technology, PSG College of \\ Technology, Coimbatore, India, TamilNadu $-641004,{ }^{4}$ Final Year, Department of Information \\ Technology, PSG College of Technology, Coimbatore, Tamil Nadu, India
}

\begin{abstract}
The COVID-19 pandemic has caused unprecedented upshot reporting around 234 million people affected worldwide. This alarming effect has led to the shortage of testing kits in many countries consequently having an impact on mortality. To combat this issue, other means for detecting the infected patients have to be put in place rather than relying on polymerase chain reaction (PCR). Radiographs are the easiest way to diagnose a patient with COVID-19 or not. This research work aims in developing a Computer-aided diagnostic system to assist radiologists in the detection of the COVID19 virus. The proposed work builds a deep learning model with Convolutional Neural Network as its baseline architecture. Due to limitations in the number of the dataset, this work uses transfer learning techniques to exploit the benefit of pre-trained models and generalize it for COVID-19 detection. Experimentation is done using 4 different models like VGG 19, MobileNet, DenseNet and InceptionResNetV2. The performance of these pre-trained models is evaluated by changing the optimizers and initializers. The covid19radiography-database dataset used for experimentation consists of 3568, 1345 and 1164 COVID-19, pneumonia and standard chest X-ray images respectively. It is observed from the experiments that the MobileNet architecture reports the highest accuracy of 99.67\% using RandomUniform initializer and Adam optimizer.
\end{abstract}

Keywords: Corona Virus Detection, Transfer Learning Techniques, MobileNet, InceptionResNetV2, COVID 19 Detection in chest X-Rays, VGG19, DenseNet, CNN.

\section{Introduction}

The WHO's global report provides a statistics of 234 million people affected and around death of 4.8 million people all around the world. Many people infected with this COVID-19 virus are asymptotic and are highly contagious. This pandemic has a devastating impact on mortality and economy though the government is taking candid steps in controlling the spread of this deadly virus. The polymerase chain reaction is used to check for the presence of the corona virus. The scarcity of testing-kits in many countries and the laborious process in analyzing the results, insufficient laboratory facilities has created a risk of contaminating the healthy individuals. Moreover timely diagnosis will save the life of many individuals. The alternate ways to diagnosis the corona virus infection is through radiography images like chest $\mathrm{X}$-rays or CT scan images [10]. Considerable time is required for the radiologists to identify the abnormalities in radiography images to detect the existence of corona virus infection. 
COVID-19 identification by radiologists requires a computer-aided diagnostic system. Deep learning models [8] are widely used in image processing to develop classification models. Deep learning models are used to learn the intrinsic feature of the images and requires little pre-processing. Deep learning minimizes the amount of human work required for feature engineering, allowing non-experts to employ deep learning models in their research. These models shows better performance for larger datasets. The dataset for COVID-19 is lesser which is not suitable for a deep learning model. In order to combat this issue, transfer learning is applied. This transfer learning model is a pre-trained model using some dataset and with the learnt features it is applied to a new dataset. The major benefits of this transfer learning is reduced training time, lesser data and improved performance [9].

The main contributions of this paper are:

- Transfer learning is applied for COVID-19 detection using VGG 19 [11], MobileNet [12], DenseNet[13] and InceptionResNetV2 [14] models; the research work also identify the model which performs better in diagnosing patient infected with corona virus

- Contrast-Enhancement is performed using CLAHE to aid in better diagnosis

- Ablation research is used to understand the architecture's influence.

- Convolution Neural Network is used as the baseline architecture for classification as COVID infected or Non-COVID individuals.

In the following order: Section 2 reviews the literature on COVID-19 diagnosis. Section 3 provides an overview of the corona virus detection architecture. Section 4 presents the experimental and analytical results. Section 5 outlines the research's findings and future scope.

\section{Literature Review}

The COVID-19 detection and classification has been of recent interests to many researchers in recent times and several works are proposed in the literature. Md Mamunur et.al [1] has presented a comparison of all pre-trained models like DenseNet, VGGNet, MobileNet and XceptionNet. Resizing and image augmentation is performed to improve the performance of the system. It is reported that the performance of VGGNet is better compared to other architectures. Osman AH, Aljahdali HM, Altarrazi SM, Ahmed A [2] uses clustering approach followed by classification the chest images as covid infected, pneumonia or non-covid. The images are labelled based on the similarities using self-organizing maps and post clustering the images are classified into one of the three classes using Locality Weighted Learning. The dataset used for analysis is collected from kaggle. Michael J. Horry et. al. [3] has used VGG 19 for transfer learning. Their experiment has been conducted on various imaging modalities like CT, X-ray and ultra sound images. The images are collected from various data sources and pre-processing techniques like resizing to match the architecture input image dimension and N-CLAHE is used for contrast enhancement. It is concluded that many deeper architectures doesn't perform well with limited dataset. The challenges in accessing publicly available dataset is also presented. Bhattarcharya et. al [4] has stated the challenges encountered in detecting covid 19 among the patients, and the role of deep learning and image processing techniques in detection. A summary of recent works in this task is listed, their data sources, imaging modalities, methodology, metrics and research challenges is presented. Aras M. Ismael and Abdulkadir S [5] has proposed a CNN architecture for covid-19 detection. Transfer learning approach is used as a feature extractor and further SVM is used for classification. The experiments were carried out by changing the kernel functions and the 
performance of the model studied. It is concluded that features extracted using ResNet 50 and a linear kernel SVM achieved the highest accuracy compared to other approaches. Ali Narin et. al. [6] has proposed a system for classifying the given x-ray images into one of the four classes as covid, non-covid, viral pneumonia, and bacterial pneumonia. Their architecture, as other systems use transfer learning approach and reports that ResNet50 found to be better in prediction compared to other models. The system is tested using a five-fold cross validation method.Tawsifur Rahman et. al. [7] has implemented various image enhancement techniques to measure the impact of it on classification of covid-19 images. Incorporating image improvement techniques such as histogram equalisation, contrast limited adaptive histogram equalisation, image complement, gamma correction, and balancing contrast equalisation. These images are given as input to the modified U-Net architecture. The authors have segmented the lung CT images and provided as input to the pre-trained networks. It is found that the segmented images aids in better system for covid-19 detection. Mustafa Ghaderzadeh and Farkhondeh Asadi [8] has presented a research review article on detecting covid 19 using deep learning approaches for various modalities. The authors has presented the various transfer learning approaches and its architectures.

\section{Proposed Architecture}

Figure 1 depicts the proposed system's general architecture. The lung images are provided as input for the architecture. Both COVID-19 infected and non-infected lung images are used in the system and each of these images are of varying sizes. The input images are subjected to pre-processing like resizing and contrast enhancement. The non-COVID infected images is of size $1024 \times 1024$ and they are resized to $256 \times 256$. Both the COVID and Non-COVID images are brought to the same dimension. CLAHE is employed for contrast enhancement to better discriminate the background and foreground images. These preprocessed images are given to a customized Convolutional Neural Network (CNN) for classifying the image as COVID infected or non-COVID infected. For smaller dataset deep learning model may not perform better and also a time-consuming task. In order to reduce the training time, transfer learning approach is used wherein the model is trained using ImageNet dataset and the features of the network is learnt and the knowledge gained is applied to the customized CNN model. The proposed work uses three different architectures namely VGG 19, MobileNet and DenseNet architecture for transfer learning. These models are tested by varying the optimizers and initializers and the performance of the system is compared.

\subsection{CLAHE}

As a result, CLAHE is an adaptation of Adaptive Histogram Equalization that uses contrast to limit the amount of amplification. You can increase contrast between similarlooking sections by adjusting the transformation function's slope.The amplification is controlled by using the clip-off strategy that depends on the neighboring regions histogram normalization and redistribution will move a fewer values above the chosen clip limit thereby ending up in effective contrast enhancement. 


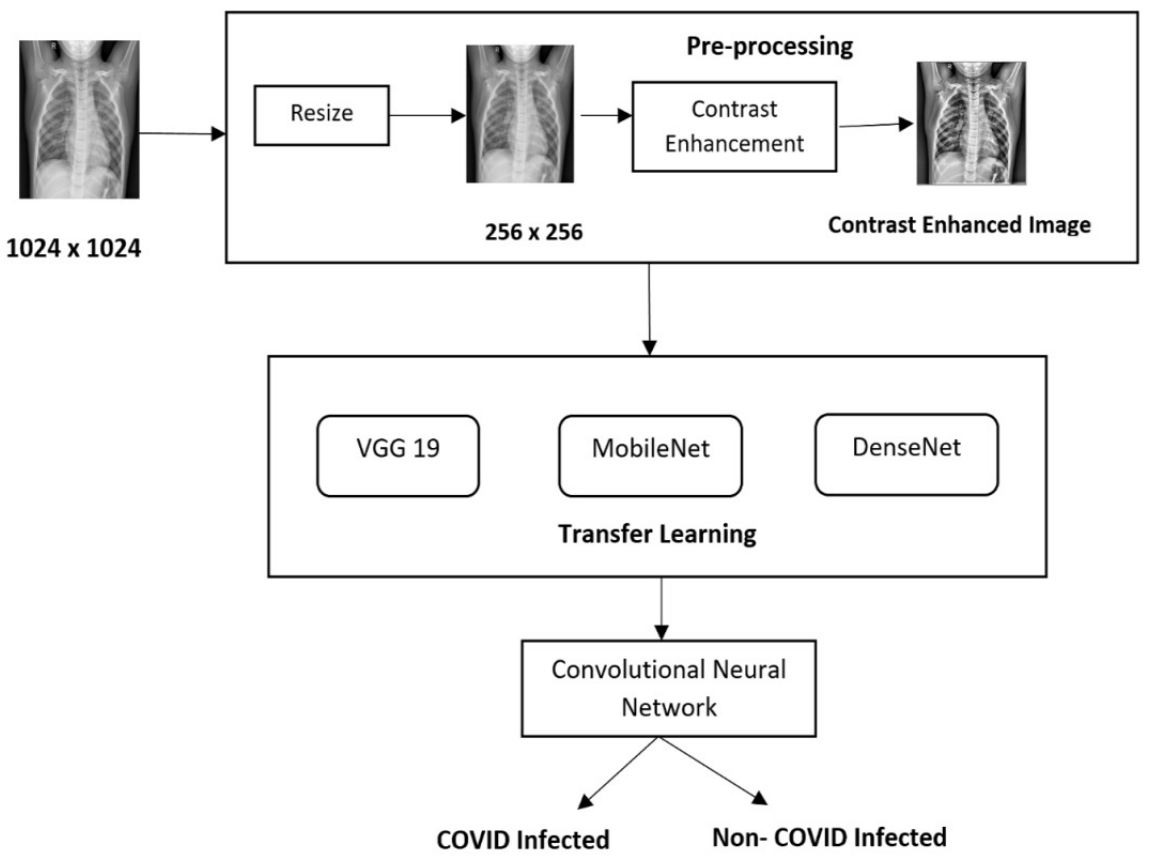

Figure 1: Architecture of the Proposed Model

\subsection{Architecture of Transfer Learning model}

Transfer learning is a method where a model is developed with a large dataset and the weights obtained for the model is applied to a new dataset. Deep learning model works better for larger dataset. If the dataset is limited transfer learning is preferred rather than training the model from scratch. In this work, VGG19, MobileNet and DenseNet are used for transfer learning and the architecture of the same is shown in Figure 2.

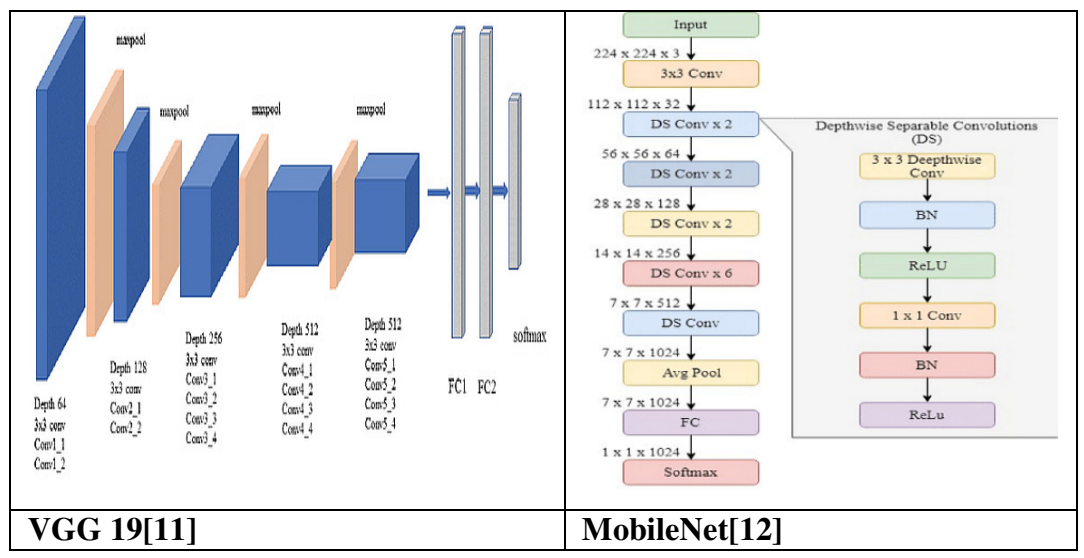




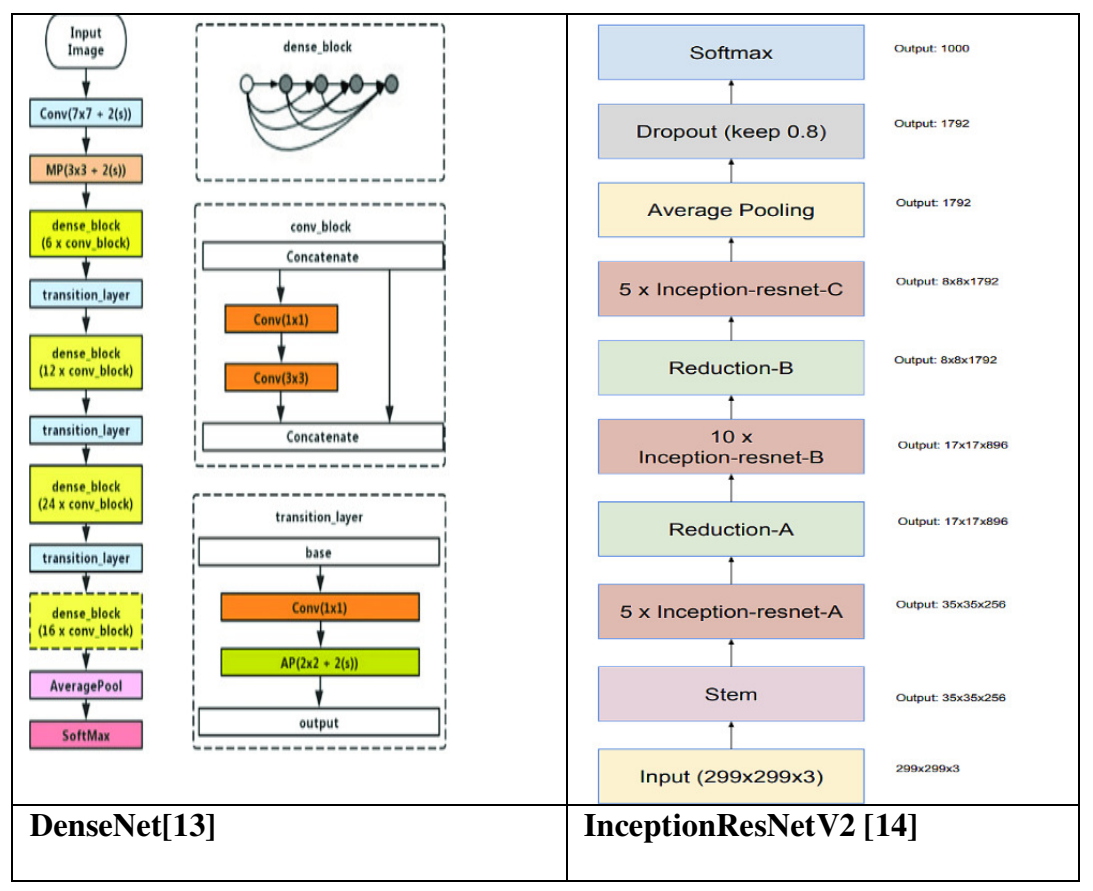

Figure 2: Architecture of Transfer Learning Approaches

\section{Experiment Analysis}

The proposed work aims in detecting whether a person is infected with covid19, pneumonia or is the individual normal. The experiments are carried using chest radiographydatabase consisting of 3568, 1345 and 1164 COVID-19, pneumonia and normal chest X-ray images respectively. The images are of dimension $256 \times 256$. The dataset is divided into training, validation and test dataset of 60:20:20 ratio. The proposed work uses transfer learning approach to identify whether a person is infected with covid-19, pneumonia or normal individual. The architectures used for transfer learning are VGG 19, MobileNet, DenseNet and InceptionResNetV2. These architectures accept images of dimension $224 \times 224$ and hence the images are resized according to this dimension to adhere to the model input dimension. Certain images required contrast enhancement which will have an improvement in the performance of the system and the same is achieved using CLAHE. Figure 3 shows the actual image and contrast enhanced chest X-ray images. 


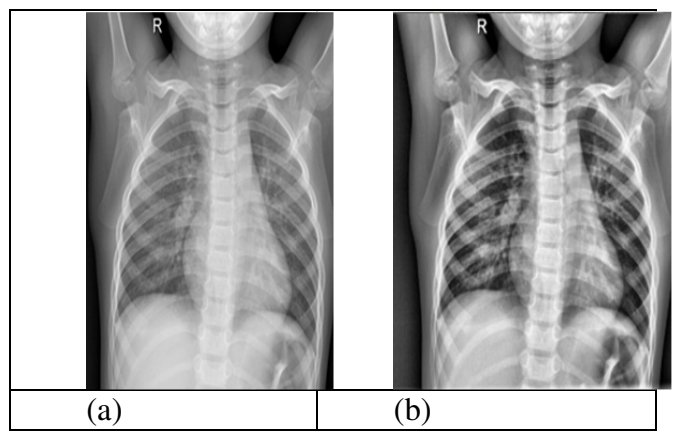

Figure 2: (a) Normal Chest X-ray (b) Contrast Enhanced Chest X-ray image

The transfer learning approach is implemented and the system is analyzed by varying the optimizers and initializers. The accuracy obtained for the architectures by changing optimizer and initializer are tabulated in Table 1.

Table 1. Accuracy for various combinations of Optimizers and Initializers

\begin{tabular}{|c|c|c|c|c|c|c|}
\hline \multirow{2}{*}{$\begin{array}{l}\text { OPTIMIZER / } \\
\text { INITIALIZER }\end{array}$} & \multicolumn{3}{|l|}{ VGG19 } & \multicolumn{3}{|l|}{ MobileNet } \\
\hline & RMSprop & SGD & Adam & RMSprop & SGD & Adam \\
\hline HeUniform & 98.67 & 95.50 & 97.67 & 98.67 & 97.83 & 98.50 \\
\hline HeNormal & 97.67 & 96.83 & 97.83 & 97.33 & 97.67 & 98.50 \\
\hline RandomUniform & 99.17 & 96.17 & 95.33 & 97.33 & 98.50 & 99.67 \\
\hline RandomNormal & 98.83 & 94.67 & 97.83 & 99.00 & 98.67 & 97.83 \\
\hline TruncatedNormal & 97.67 & 96.83 & 98.83 & 97.50 & 97.83 & 98.33 \\
\hline \multirow[b]{2}{*}{$\begin{array}{l}\text { OPTIMIZER / } \\
\text { INITIALIZER }\end{array}$} & \multicolumn{3}{|l|}{ DenseNet } & \multicolumn{3}{|c|}{ InceptionResNetV2 } \\
\hline & RMSprop & SGD & Adam & RMSprop & SGD & Adam \\
\hline HeUniform & 98.17 & 97.83 & 96.83 & 88.17 & 82.67 & 86.16 \\
\hline HeNormal & 98.33 & 97.33 & 97.67 & 87.13 & 87.33 & 87.13 \\
\hline RandomUniform & 99.00 & 97.50 & 97.00 & 86.00 & 87.50 & 86.19 \\
\hline RandomNormal & 97.33 & 96.33 & 98.33 & 83.33 & 86.33 & 88.37 \\
\hline TruncatedNormal & 98.50 & 97.33 & 96.67 & 83.50 & 87.33 & 85.67 \\
\hline
\end{tabular}

The precision, recall and the loss for various transfer learning approaches are presented in Figure 3. It has been observed that the MobileNet performs better compared to other transfer learning approaches when RandomUniform initializer and Adam optimizer. The loss function used is categorical cross entropy. 


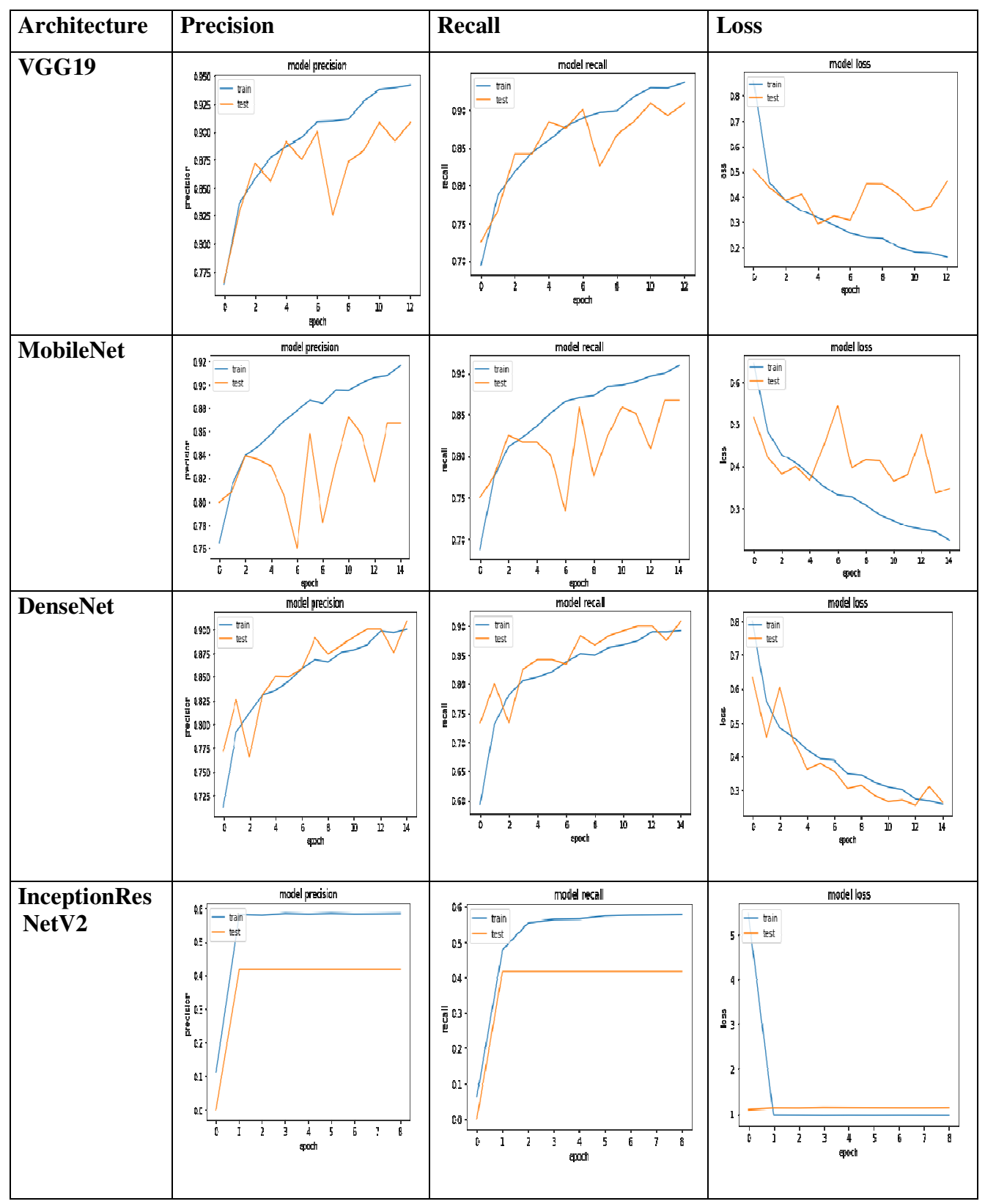

Figure 3: Precision, Recall and Loss for the transfer learning approaches 


\section{Conclusion}

The proposed system detects the given chest $\mathrm{x}$-ray images as covid, non-covid or normal images. The experimental analysis is done using the chest $\mathrm{x}$-ray radiography images from kaggle. The images used for training, validation and test are in the ratio of 60:20:20. The proposed system resizes the image into $224 \times 224$ dimension and Contrast Limited Adaptive Histogram Equalization is used for contrast enhancement. The pre-processed images are given to the system for classifying the images to one of the classes. Four transfer learning approaches VGG19, MobileNetV2, DenseNet201 and InceptionResNetV2 are analysed and is found that MobileNetV2 performs better with RandomUniform initializer and Adam optimizer. Semantic segmentation can be performed to improve the performance of the system.

\section{References}

[1] Md Mamunur Rahaman et.al, "Identification of COVID-19 samples from chest X-Ray images using deep learning: A comparison of transfer learning approaches", Journal of X-Ray Science and Technology, Vol: 28, pp: 821-839, 2020.

[2] Osman AH, Aljahdali HM, Altarrazi SM, Ahmed, "A SOM-LWL method for identification of COVID-19 on chest X-rays”, PLoS ONE 16(2): e0247176, 2021. https://doi.org/10.1371/ journal.pone. 0247176

[3] Michael J Horry et.al., "COVID-19 Detection Through Transfer Learning Using Multimodal Imaging Data", IEEE Access, Vol: 8, pp:149808-149824,2020. Doi: 10.1109/ACCESS.2020.3016780.

[4] Sweta Bhattacharya et.al, "Deep learning and medical image processing for coronavirus (COVID19) pandemic: A survey", Sustainable Cities and Society 65, pp: 1-18, 2021.

[5] Aras M. Ismael a , Abdulkadir S, engür, "Deep learning approaches for COVID-19 detection based on chest X-ray images", Expert Systems With Applications, 164, 2021. https://doi.org/10.1016/j.eswa.2020.114054

[6] Ali Narin, Ceren Kaya, and Ziynet Pamuk, "Automatic detection of coronavirus disease (COVID-19) using X-ray images and deep convolutional neural networks", Pattern Analysis and Applications, Vol: 24, pp: 1207-1220, 2021. https://doi.org/10.1007/s10044-021-00984-y

[7] Tawsifur Rahman et.al., "Exploring the effect of image enhancement techniques on COVID-19 detection using chest X-ray images", Computers in Biology and Medicine, vol: 132, 104319, 2021. https://doi.org/10.1016/j.compbiomed.2021.104319

[8] Mustafa Ghaderzadeh and Farkhondeh Asadi, "Deep Learning in the Detection and Diagnosis of COVID-19 Using Radiology Modalities: A Systematic Review", Journal of Healthcare Engineering, Vol: 2021, pp: 1-10, 2021. https://doi.org/10.1155/2021/6677314

[9] Weiss,K., Khoshgoftaar, T.M. \& Wang, D, “A survey of transfer learning”, Journal of Big Data 3, 9, 2016. https://doi.org/10.1186/s40537-016-0043-6

[10] Jeffrey P. Kanne et. al., "COVID-19 Imaging: What We Know Now and What Remains Unknown”, Radiology, Vol: 299, pp:E262-E279, 2021. https://doi.org/10.1148/radiol.2021204522

[11] Karen Simonyan and Andrew Zisserman, "Very Deep Convolutional Networks For Large-Scale Image Recognition", In proceedings of ICLR 2015, pp:1-14, 2015.

[12] Andrew G. Howard et. al., "MobileNets: Efficient Convolutional Neural Networks for Mobile Vision Applications", Computer Vision and Pattern Recognition, pp:1-9, 2017.

[13] Gao Huang, Zhuang Liu, Laurens van der Maaten and Kilian Q. Weinberger, "Densely Connected Convolutional Networks", In Proceedings of CVPR 2017, pp: 1-9, 2017. 
[14] Christian Szegedy, Sergey Ioffe, Vincent Vanhoucke, Alex Alemi, "Inception-v4, InceptionResNet and the Impact of Residual Connections on Learning", In Proceedings of Computer Vision and Pattern Recognition 2016, pp: 1-12, 2016. 